\title{
Prospective, Randomized, Multicenter, Controlled Trial of a Bioartificial Liver in Treating Acute Liver Failure
}

\author{
Achilles A. Demetriou, MD, PhD, * Robert S. Brown, Jr, MD, MPH, † Ronald W. Busuttil, MD, PhD, $\neq$ \\ Jeffrey Fair, MD, § Brendan M. McGuire, MD, 9 Philip Rosenthal, MD,\| \\ Jan Schulte Am Esch, II, MD, ** Jan Lerut, MD, †† Scott L. Nyberg, MD, PhD,tf \\ Mauro Salizzoni, MD, §£ Elizabeth A. Fagan, MD 19 Bernard de Hemptinne, MD,||| \\ Christoph E. Broelsch, MD, PhD,*** Maurizio Muraca, MD, PhD, $\uparrow \dagger$

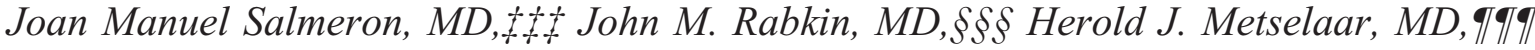 \\ Daniel Pratt, MD, ||||| Manuel De La Mata, MD,**** Lawrence P. McChesney, MD, ††† \\ Gregory T. Everson, MD,t+ft Philip T. Lavin, PhD, $\$ \oint \oint \oint$ Anthony C. Stevens, MD, 1999 \\ Zorina Pitkin, PhD, 9999 and Barry A. Solomon, PhD 1999
}

Objective: The HepatAssist liver support system is an extracorpo-
real porcine hepatocyte-based bioartificial liver (BAL). The safety
and efficacy of the BAL were evaluated in a prospective, random-

From the *Liver Support Unit, Department of Surgery, Cedars-Sinai Medical Center, Los Angeles, CA; †Center for Liver Disease and Transplantation, New York Presbyterian Hospital, New York, NY; †UCLA Transplant Center, University of California, Los Angeles, CA; §Division of Abdominal Transplant Surgery, University of North Carolina at Chapel Hill, Chapel Hill, NC; qUniversity of Alabama Liver Center, University of Alabama at Birmingham, Birmingham, AL; |Liver Transplant Program, University of California, San Francisco Medical Center, San Francisco, CA; **Department of Hepatobiliary Surgery, University Hospital HamburgEppendorf, Hamburg, Germany; $\dagger \dagger$ Cliniques Universitaires Saint Luc, Brussels, Belgium; +\$Mayo Medical School, Liver Transplant Unit, Rochester,

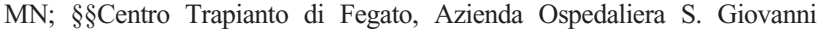
Battista, Torino, Italy; qqSection of Hepatology, Rush-Presbyterian-St Luke's Medical Center, Chicago, IL; |||Universitair Zeikenhaus Gent, Gent, Belgium; ***Department of General and Transplantation Surgery, University of Essen, Essen, Germany; †† Sciences, University of Padova, Padova, Italy; $+\$+t$ Liver Unit, Intensive Care Unit, Hospital Clinic Provincial de Barcelona, Barcelona, Spain; $\$ \S \S$ Liver Transplant Program, Oregon Health \& Sciences University, Portland, OR; qศףLiver Transplant, Department of Hepatogastroenterology, Erasmus University Medical Center, Rotterdam, The Netherlands; |||||New England Medical Center, Division of Gastroenterology, Boston, MA; ****Liver Unit of the Digestive Disease Service, University of Cordoba, Cordoba, Spain; $+\dagger \dagger$ Division of Transplant Surgery, Medical College of Ohio, Toledo, OH; It+Hepatology/Liver Transplantation, University of Colorado Health Sciences Center, Denver, CO; $\S \S \S \S A v e r i o n$, Inc., Framingham, MA; and \$19 9 Circe Biomedical, Inc, Lexington, MA.

Reprints: Achilles A. Demetriou, MD, PhD, Department of Surgery, CedarsSinai Medical Center, 8700 Beverly Blvd, Suite 8215, North Tower, Los Angeles, CA 90048. E-mail: demetriou@csmc.edu.

Copyright (C) 2004 by Lippincott Williams \& Wilkins

ISSN: 0003-4932/04/23905-0660

DOI: $10.1097 / 01$. sla.0000124298.74199.e5 ized, controlled, multicenter trial in patients with severe acute liver failure.

Summary Background Data: In experimental animals with acute liver failure, we demonstrated beneficial effects of the BAL. Similarly, Phase I trials of the BAL in acute liver failure patients yielded promising results.

Methods: A total of 171 patients ( 86 control and 85 BAL) were enrolled. Patients with fulminant/subfulminant hepatic failure and primary nonfunction following liver transplantation were included. Data were analyzed with and without accounting for the following confounding factors: liver transplantation, time to transplant, disease etiology, disease severity, and treatment site.

Results: For the entire patient population, survival at 30 days was $71 \%$ for BAL versus $62 \%$ for control $(P=0.26)$. After exclusion of primary nonfunction patients, survival was $73 \%$ for BAL versus $59 \%$ for control $(\mathrm{n}=147 ; P=0.12)$. When survival was analyzed accounting for confounding factors, in the entire patient population, there was no difference between the 2 groups (risk ratio $=0.67 ; P=$ 0.13 ). However, survival in fulminant/subfulminant hepatic failure patients was significantly higher in the BAL compared with the control group (risk ratio $=0.56 ; P=0.048$ ).

Conclusions: This is the first prospective, randomized, controlled trial of an extracorporeal liver support system, demonstrating safety and improved survival in patients with fulminant/subfulminant hepatic failure.

(Ann Surg 2004;239: 660-670)

C urrently, the standard treatment of acute liver failure (ALF) involves supportive care that focuses on bridging patients to either transplantation or spontaneous recovery. Orthotopic liver transplantation is the only current therapy shown to improve patient survival. Several extracorporeal 
liver support systems have been used to treat patients with ALF attempting to either bridge them to recovery or to transplantation. These include cell-based and non-cell-based systems. Non-cell-based techniques (eg, high-volume plasma exchange and albumin dialysis), initially developed for other conditions, have been used to treat severe acute liver failure for more than a decade. No controlled, multicenter, large, randomized, prospective trials have been carried out using non-cell-based systems; therefore, their effect on survival remains unknown. ${ }^{1,2}$

Extracorporeal liver perfusion using whole human and pig livers has been shown to effectively support patients with ALF for several days, ${ }^{3}$ but it is impractical for wider use because of limited availability of human livers and lack of quality control and consistency for animal livers. As a result, several extracorporeal cell-based devices were developed. Early Phase I studies have been performed using whole blood or plasma perfusion through cartridges (mostly hollow-fiber bioreactors) containing either human hepatoblastoma (HepG2-C3A) cells or freshly isolated porcine hepatocytes. The devices appeared to be well tolerated by the patients, but these studies did not demonstrate a survival advantage over standard of care in appropriately controlled settings. ${ }^{4}$

A bioartificial liver (BAL), developed by the group at Cedars-Sinai Medical Center using cryopreserved porcine hepatocytes, is the most extensively studied of the extracorporeal cell-based liver support systems. Several preliminary uncontrolled clinical studies examining the effect of this therapy in patients with ALF have shown that BAL treatment improved neurologic function, reduced intracranial pressure (ICP), and increased cerebral perfusion pressure.$^{5-7}$ Here, we report the results of the first Phase II/III prospective, randomized, multicenter, controlled trial examining the effect of BAL treatment on survival in patients with ALF. This is the largest prospective, randomized, controlled, multicenter trial of any artificial liver support system to date.

\section{METHODS}

\section{BAL}

The BAL is comprised of porcine hepatocytes (7 billion) housed within a hollow-fiber bioreactor (Fig. 1). Cell isolation and cryopreservation methods as well as subsequent cell thawing and processing techniques have been described elsewhere. ${ }^{5,8,9}$ Cryopreservation allows long-term storage as well as complete safety assessment before clinical use of the cells. Additionally, the system includes a perfusion pump, a charcoal column, a combined oxygenator/blood warmer, and custom-tubing that connects the various components to a commercially available plasmapheresis machine. ${ }^{6}$

During a BAL treatment, plasmapheresis is performed via a femoral vein double-lumen catheter. The separated plasma is subsequently pumped into the BAL that continu-

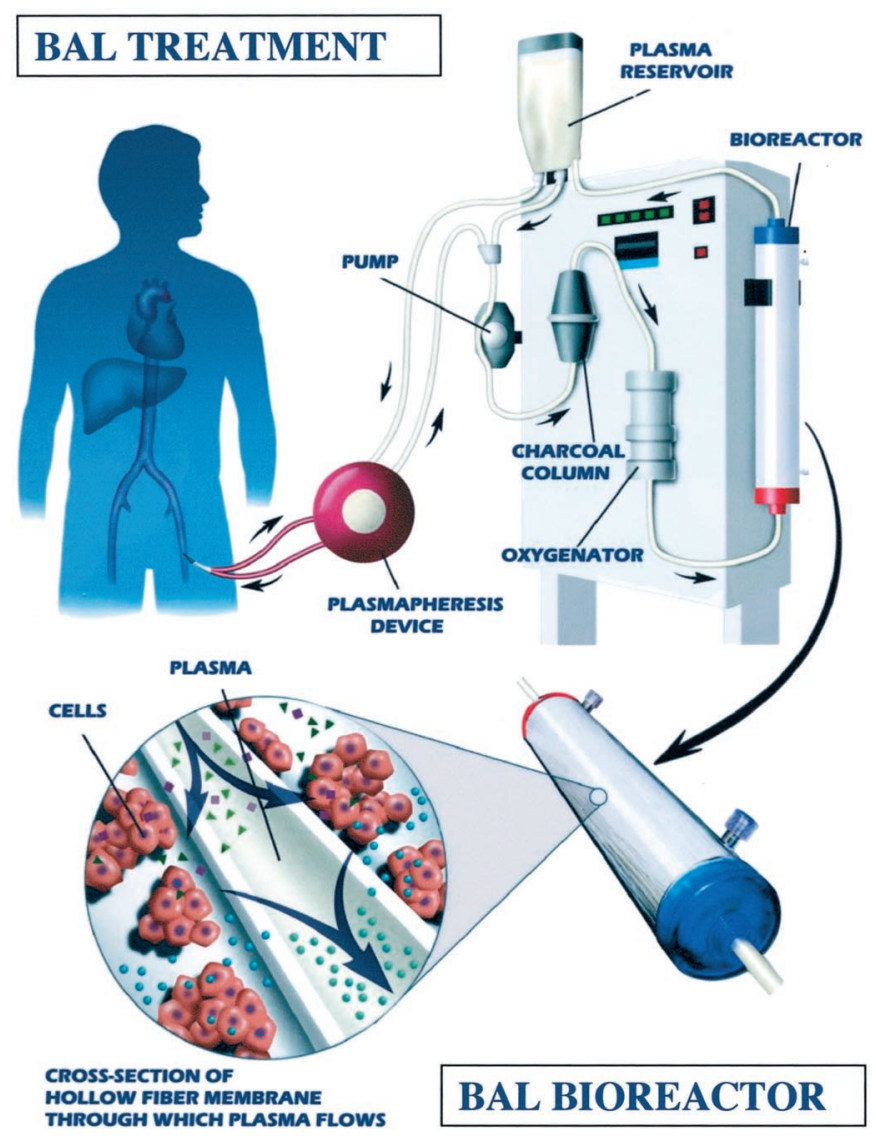

FIGURE 1. Schematic outline of the BAL and the bioreactor cartridge loaded with microcarrier-attached porcine hepatocytes.

ously circulates the plasma through the BAL hollow fiber device and other components of the BAL system. The plasma flows through the lumens of the fibers that are surrounded by a suspension of porcine hepatocytes. The pore size of the membrane $(0.15 \mu \mathrm{m})$ prevents hepatocytes and cell debris from passing through the fiber wall and entering a patient's bloodstream.

\section{Study Design}

This is a prospective, randomized, multicenter, controlled trial that evaluates the safety and efficacy of BAL treatment on survival in patients with ALF. ALF patients had fulminant hepatic failure (FHF)/subfulminant hepatic failure (SHF), or primary nonfunction (PNF) following orthotopic liver transplantation. Eligible subjects were randomized 1:1 into either the standard of care control group or the BAL treatment plus standard of care BAL group. Randomization and group assignment were carried out centrally using an automated phone system. Subjects were stratified according to etiology (viral vs. acetaminophen vs. other/indeterminate 
vs. primary nonfunction postliver transplantation), investigational site, and stage of hepatic encephalopathy. ${ }^{10,11}$

Patients in the BAL group underwent a treatment of 6 hours (preceded by 1 hour of closed plasmapheresis to ensure hemodynamic stability) daily as tolerated. The treatment number was variable but limited to 14 . Treatment was terminated if an organ became available for transplantation, if there was significant clinical improvement, if the patient experienced marked deterioration, if there was a significant adverse event, or if the patient died. Patients in the control group received intensive critical care according to current standard best practices at each study site. All subjects were assessed for clinical status every 12 hours and seen for follow-up visits at $1,2,3,6,9$, and 12 months during the post-treatment period.

Safety and efficacy data were blinded to the sponsor (Circe Biomedical, Inc., Lexington, MA) and the other clinical sites. Outcomes were monitored by a Data and Safety Monitoring Board (DSMB).

\section{Patients}

Patients were enrolled at 11 U.S. and 9 European sites during a 3-year period. Study inclusion criteria included weight greater than $40 \mathrm{~kg}$ and stage III or IV hepatic encephalopathy for patients with FHF/SHF. PNF patients were enrolled if all of the following were present: more than 48 hours and less than 7 days after liver transplantation, listed as highest priority for retransplantation, lack of bile production if a T-tube was present, presence of blood flow through the liver by ultrasound/Doppler, elevated aspartate serum transferase ( $>2500 \mathrm{IU})$, prothrombin time ( $>16.5$ seconds) or both, and lack of posttransplant improvement in liver function. Exclusion criteria included presence of chronic liver disease, pregnancy, known hypersensitivity to porcine products, and other criteria similar to contraindications for transplantation (ie, sepsis, severe cardiac disease, uncontrolled bleeding, and evidence of irreversible brain damage).

Informed consent was obtained from each patient's next of kin/legal guardian, and the study protocol conformed to the ethical guidelines of the 1975 Declaration of Helsinki as reflected in a priori approval by the institutional review committees at participating institutions.

\section{Data Analysis}

The primary end point of the study was patient survival. It was analyzed for the following populations: all study patients, FHF/SHF and PNF ( $\mathrm{n}=171)$; and FHF/SHF patients only $(\mathrm{n}=147)$.

Patient survival data were analyzed using 2 methods:

\section{Thirty-Day Survival}

Thirty-day survival postrandomization, with or without transplantation, was examined. This analysis was performed at various predetermined time points during the trial based on the number of patients accrued.

The protocol-defined statistical analysis plan was based on the Whitehead Triangular Test, ${ }^{12,13}$ in which stopping rules related to safety, efficacy, and futility determined study termination. The stopping rules used by the DSMB were modeled after an overall 30-day survival rate of $70 \%$ for the control group and $86 \%$ for the BAL group. Thus, the total number of patients to be enrolled in the study was not predetermined, but theoretical maximum enrollment was 404 patients.

\section{Cox Proportional Hazard Model}

Given the small sample size and nonblinded nature of the study, differences in mean time to transplant, which could bias the interpretation of 30-day survival, were addressed by using a covariate time-dependent proportional hazard model, which controlled for transplantation and the time to transplantation as well as other confounding factors including treatment site, severity of illness (level of encephalopathy), and disease etiology. The proportional hazard model used risk ratio $(\mathrm{RR})$ to describe treatment effects while controlling for transplantation as well as other confounding factors as time-varying covariates.

Other secondary end points examined in this study included "time to death" and "time to transplantation," liver and renal function tests, metabolic, hematological, and hemodynamic parameters, as well as neurologic assessment. ICP monitors were used only in a few participating centers and were placed at various time points following patient enrollment.

\section{Safety}

All patients were monitored for occurrence of adverse events from the time of entry into the study through 1-year postrandomization. In addition, blood samples from all enrolled subjects were tested for the presence of porcine endogenous retrovirus (PERV) by the Centers for Disease Control (Atlanta, GA). ${ }^{14}$ Baseline blood samples were collected for all subjects following randomization. Additional samples were collected for the BAL group at the end of the treatment period and at 1, 2, 3, 6, 9, and 12 months postrandomization. Presence of PERV was tested by 2 methods: 1) serologic screen to detect antibodies to PERV and 2) polymerase chain reaction analysis of peripheral blood lymphocytes to detect PERV DNA sequences. ${ }^{14,15}$

\section{RESULTS}

\section{Patient Demographics}

A total of 171 patients with ALF were enrolled in this study. This included 24 PNF patients following transplantation and 147 patients with FHF/SHF (121 FHF patients and 
26 SHF patients in stage III or IV hepatic encephalopathy). Patients were randomly assigned either to the control ( $\mathrm{n}=$ $86)$ or to the BAL group $(n=85$; Table 1$)$. Etiologies within the FHF/SHF patient population included known causes such as viral hepatitis and acetaminophen and other drug or chemical toxicities ( $\mathrm{n}=83 ; 43$ control and $40 \mathrm{BAL})$, as well as other indeterminate etiologies $(\mathrm{n}=64 ; 31$ control and 33 BAL). Of the total patients with ALF, 51 (29.8\%) were men and $120(70.2 \%)$ were women, with a mean age of 37 years (range, 10-69 years). The demographic characteristics of the patients are summarized in Table 2. The control and BAL groups were well balanced.

The number of BAL treatments ranged from 1 to 9 (mean 2.9) per patient. The primary reason for termination of treatment was successful procurement of a liver for transplantation. BAL treatment was terminated prior to transplantation. Five patients in the BAL group never received a BAL treatment but were included in the analysis on an "intentionto-treat basis."

\section{Safety}

Most adverse events occurred during the first 30 days postrandomization and were quite common as expected in patients who were critically ill at study entry. Adverse events were balanced across the control and BAL groups in both incidence and severity (Table 3). Thrombocytopenia occurred with the highest incidence in both the control and BAL groups $(33.7 \%$ and $38.8 \%$, respectively). Although more instances of renal failure (15.1\% control, $7.1 \% \mathrm{BAL})$ and high ICP (11.6\% control, 7.1\% BAL) were reported in the control group, the differences were not statistically significant. All patients tested in this study were negative for the presence of PERV infection at all time points. ${ }^{14}$

\section{Efficacy Analysis}

\section{Primary End Point}

\section{Thirty-Day Survival}

Thirty-day survival in the overall study population $(\mathrm{n}=171)$ was $71 \%$ in the BAL group and $62 \%$ in the control

TABLE 1. Stages of Hepatic Encephalopathy

\begin{tabular}{lccc}
\hline $\begin{array}{l}\text { Stage of Hepatic } \\
\text { Encephalopathy }\end{array}$ & $\begin{array}{c}\text { Control } \\
(\mathbf{N}=\mathbf{8 6}) \\
{[\mathbf{n}(\mathbf{\%})]}\end{array}$ & $\begin{array}{c}\text { BAL } \\
(\mathbf{N}=\mathbf{8 5}) \\
{[\mathbf{n}(\mathbf{\%})]}\end{array}$ & $\begin{array}{c}\text { Total } \\
(\mathbf{N}=\mathbf{1 7 1}) \\
{[\mathbf{n}(\mathbf{\%})]}\end{array}$ \\
\hline III & $31(36)$ & $33(39)$ & $64(37)$ \\
IV & $28(33)$ & $25(29)$ & $53(31)$ \\
Not evaluable* & $15(17)$ & $15(18)$ & $30(18)$ \\
PNF & $12(14)$ & $12(14)$ & $24(14)$ \\
\hline
\end{tabular}

*Usually not evaluable because of drug-induced coma.

BAL, bioartificial liver; PNF, primary nonfunction following transplantation.
TABLE 2. Patient Demographics

\begin{tabular}{lcc}
\hline Patient Characteristic & Control [n (\%)] & BAL $[\mathbf{n}(\%)]$ \\
\hline Gender & $26(30.2)$ & $25(29.4)$ \\
Male & $60(69.8)$ & $60(70.6)$ \\
Female & 86 & 85 \\
Total & & \\
Race & $64(74.4)$ & $54(63.5)$ \\
White & $7(8.1)$ & $15(17.6)$ \\
Black & $6(7.0)$ & $7(8.2)$ \\
Asian & $6(7.0)$ & $5(5.9)$ \\
Hispanic & $3(3.5)$ & $4(4.7)$ \\
Other & 86 & 85 \\
Total & & 85 \\
Age (yr) & 86 & 10 \\
n & 10 & 69 \\
Minimum & 66 & 37.2 \\
Maximum & 37.3 & 14.1 \\
Mean & 14.7 & \\
Standard Deviation & &
\end{tabular}

BAL, bioartificial liver.

group $(P=0.26$; Table 4). Survival in the FHF/SHF group was higher in BAL-treated patients. However, the difference in survival was not statistically significant. The 30 -day survival data are presented in Table 4. The DSMB terminated the trial based on these results because it determined that trial continuation, under the protocol in place and using this type of data analysis, was likely to be futile for the primary end point of 30-day survival.

\section{Cox Proportional Hazard Model}

Additional data analysis was performed which took into account several confounding factors: liver transplantation,

TABLE 3. Common Adverse Events*

\begin{tabular}{lcc}
\hline Common Adverse Event & $\begin{array}{c}\text { Control } \\
{[\mathbf{n}(\%)]}\end{array}$ & $\begin{array}{c}\text { BAL } \\
{[\mathbf{n}(\%)]}\end{array}$ \\
\hline Thrombocytopenia & $29(33.7)$ & $33(38.8)$ \\
Hypotension & $12(14.0)$ & $12(14.1)$ \\
Hypoxia & $6(7.0)$ & $9(10.6)$ \\
Renal failure & $13(15.1)$ & $6(7.1)$ \\
Graft rejection & $7(8.1)$ & $11(12.9)$ \\
Increased intracranial pressure & $10(11.6)$ & $6(7.1)$ \\
Sepsis & $10(11.6)$ & $9(10.6)$ \\
Pulmonary edema & $6(7.0)$ & $6(7.1)$ \\
Acute renal failure & $4(4.7)$ & $5(5.9)$ \\
Brain herniation & $5(5.8)$ & $4(4.7)$ \\
\hline
\end{tabular}

*Common adverse events are presented up to day 30 . BAL, bioartificial liver. 
TABLE 4. Thirty-Day Survival in Acute Liver Failure

\begin{tabular}{lrrrr}
\hline Etiology & n & $\begin{array}{c}\text { Control* } \\
{[\mathbf{n}(\%)]}\end{array}$ & $\begin{array}{c}\text { BAL* } \\
{[\mathbf{[ n}(\%)]}\end{array}$ & $\boldsymbol{P}$ Value \\
\hline All patients & 171 & $53 / 86(62)$ & $60 / 85(71)$ & 0.259 \\
FHF/SHF & 147 & $44 / 74(59)$ & $53 / 73(73)$ & 0.117 \\
PNF & 24 & $9 / 12(75)$ & $7 / 12(58)$ & 0.667
\end{tabular}

*Survivors/total patients.

FHF, fulminant hepatic failure; SHF, subfulminant hepatic failure; PNF, primary nonfunction post-transplantation; BAL, bioartificial liver.

time to transplantation, disease etiology, disease severity, and treatment site. When examining the impact of transplantation on survival in the entire patient population using this model, the RR for transplantation in the first 30 days was $0.31(P=$ $0.0001)$. The majority of patients $(55 \%)$ underwent transplantation in the first 30 days following randomization. Survival for all transplanted patients was $89 \%$ for the BAL group and $80 \%$ for the control group compared with $50 \%$ and $38 \%$, respectively, in those not transplanted (Table 5).

We used the covariate time-dependent proportional hazard model to analyze patient survival to account primarily for the powerful impact of transplantation on patient survival. The RR for BAL treatment in all ALF patients $(\mathrm{n}=171)$ was 0.67 . This was not statistically significant $(P=0.13)$. However, the RR for BAL treatment in patients with FHF/SHF $(\mathrm{n}=147)$ was 0.56 . This finding was statistically significant $(P=0.048)$.

\section{Secondary End Points}

\section{Time to Death}

Figure $2 \mathrm{~A}$ demonstrates that there was no statistically significant prolongation of survival in the BAL group in the FHF/SHF patient populations $(P=0.10)$. In the FHF/SHF group, patients with known etiologies $(\mathrm{n}=83)$, a significant difference $(P=0.009)$ in time to death within the first 30 days was noted (Fig. 2B); no significant effect was seen in

TABLE 5. Survival Rates of Transplanted Versus

Nontransplanted Patients

\begin{tabular}{lccc}
\hline Group & $\begin{array}{c}\text { Control* } \\
{[\mathbf{n}(\%)]}\end{array}$ & $\begin{array}{c}\text { BAL* } \\
{[\mathbf{n}(\%)]}\end{array}$ & $\boldsymbol{P}$ Value \\
\hline $\begin{array}{l}\text { Transplanted patients } \\
\quad(\mathrm{N}=94)\end{array}$ & $39 / 49(80)$ & $40 / 45(89)$ & 0.22 \\
$\begin{array}{l}\text { Nontransplanted patients } \\
(\mathrm{N}=77)\end{array}$ & $14 / 37(38)$ & $20 / 40(50)$ & 0.38 \\
\hline
\end{tabular}

*Survivors/total patients.

BAL, bioartificial liver. patients with FHF/SHF due to other/indeterminate etiologies (data not shown).

\section{Time to Transplantation}

The mean time interval between randomization and transplantation was longer for BAL patients (5 days) than control patients (3 days); this, however, was not statistically significant $(P=0.21)$.

\section{Laboratory Values and Neurologic Outcomes}

The mean total serum bilirubin levels in BAL patients, from day 4 through day 9 postrandomization, were significantly lower than those of control patients in the entire patient population (Fig. 3). No statistically significant differences were noted in other laboratory values (hematological, metabolic), neurologic assessment, or hemodynamic parameters in the entire patient population.

\section{DISCUSSION}

Multiple attempts have been made over the years to develop an effective liver support treatment of ALF. No major prospective, appropriately randomized, multicenter, trials have been carried out. Performing such trials is difficult because ALF patients are aggressively treated in intensive care units and receive maximal standard pharmacologic, physiologic, and other interventions to provide metabolic, respiratory, and hemodynamic support. This makes measurements of metabolic parameters, biochemical markers, and neurologic assessment problematic due to the number and complexity of interventions. In addition, there still exists significant variation in the standard of care among treatment centers. As an example, some centers routinely use freshfrozen plasma to correct coagulopathy, whereas others do not; ICP monitoring is not uniformly used across treatment sites.

ALF is not a homogeneous disease. Disease etiology and severity are important clinical variables that determine outcome. PNF is a syndrome in which intracranial hypertension is not usually a major clinical management issue. Patients with FHF are more likely to have significant intracranial hypertension and cerebral edema and often need urgent transplantation. SHF patients may have a more prolonged and subtle course, but the disease is associated with high mortality due to sepsis and multiorgan failure after prolonged stay in an intensive care unit. Acetaminophen-induced FHF can be associated with high spontaneous recovery rate; however, patients with severe variants of the disease can rapidly progress to cerebral edema and death.

Liver transplantation is the most important predictor of survival, and this was also demonstrated in this study population. When patients are transplanted early in the course of their disease, it makes demonstration of a survival advantage in the treatment group difficult because simply there is not 
A

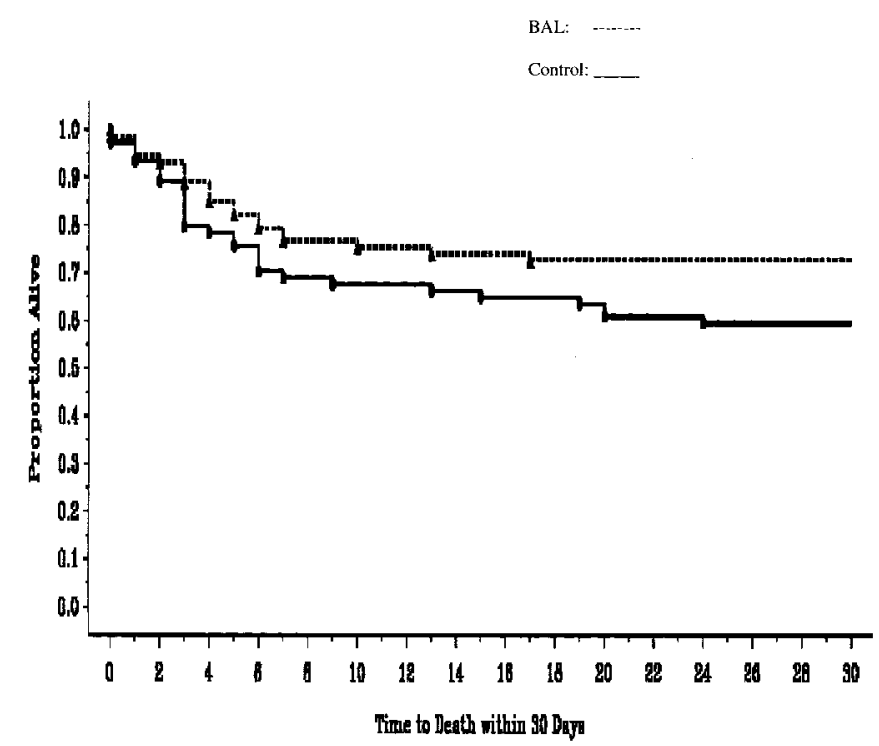

B

BAL

Control

FIGURE 2. A: Time to death in FHF/ SHF patients $(n=147 ; P=0.10)$. B: Time to death in patients with FHF/ SHF of known etiology $(\mathrm{n}=83 ; \mathrm{P}=$ 0.009).

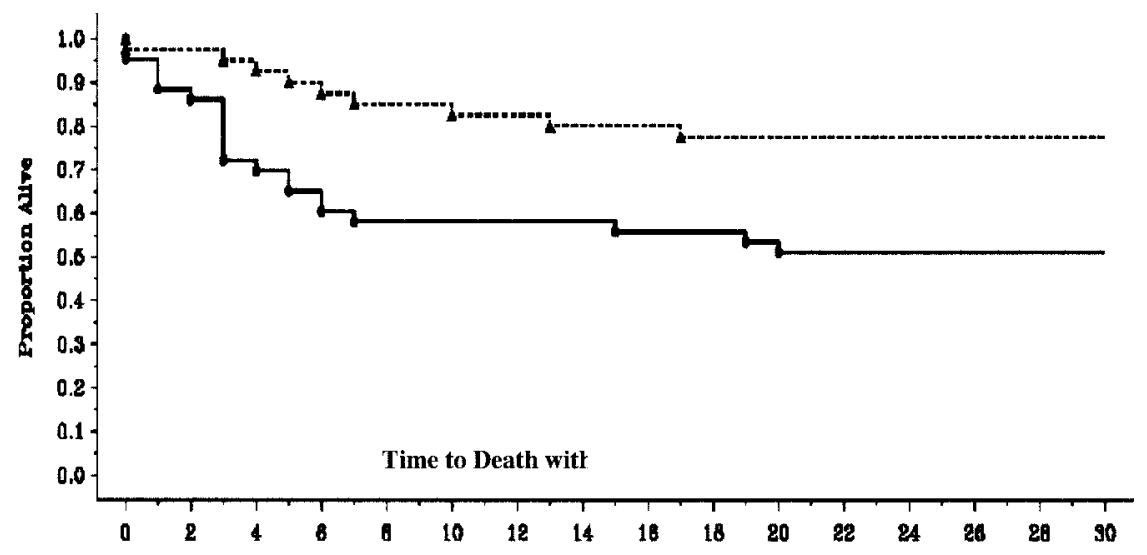

enough time to demonstrate BAL efficacy in a setting where patients are receiving full supportive care. This is particularly true for PNF patients because of the urgency associated with securing a liver for retransplantation in such patients. Another factor complicating this trial was a change in national organ allocation policy for patients with severe ALF in the United States after the trial was initiated. This resulted in more organs being allocated for these patients and shortening of transplant waiting periods for patients with severe ALF. Liver transplantation was a dominant factor in determining patient survival, as evidenced by an overall survival rate of $84 \%$ after transplantation and as high as $92 \%$ in transplanted FHF/SHF patients, which was greater than the 1-year survival of transplanted patients reported in the United States at the time of enrollment (Table 5). ${ }^{16}$ Therefore, differences in "time to transplantation" between the groups confounded the results.
This view is supported by a meta-analysis of the short-term survival of ALF patients receiving standard medical care with and without liver transplantation. ${ }^{16}$

No significant survival advantage was noted for BALtreated patients when 30-day survival was examined without taking into account confounding factors. The Whitehead Triangular Test was used with predetermined stopping boundaries assuming a 30-day survival of between $70 \%$ in the control group and $86 \%$ in the BAL group based upon Phase I trial results at the time the study was designed. This analytical model was used because it provided a statistical test to minimize the number of patients required for success. However, it also led to higher likelihood of study failure due to futility after a small number of patients were enrolled. The trial was terminated because it was concluded that demonstration of a significant survival benefit using this method of 


\section{- Control \\ $\Delta \mathrm{BAL}$}

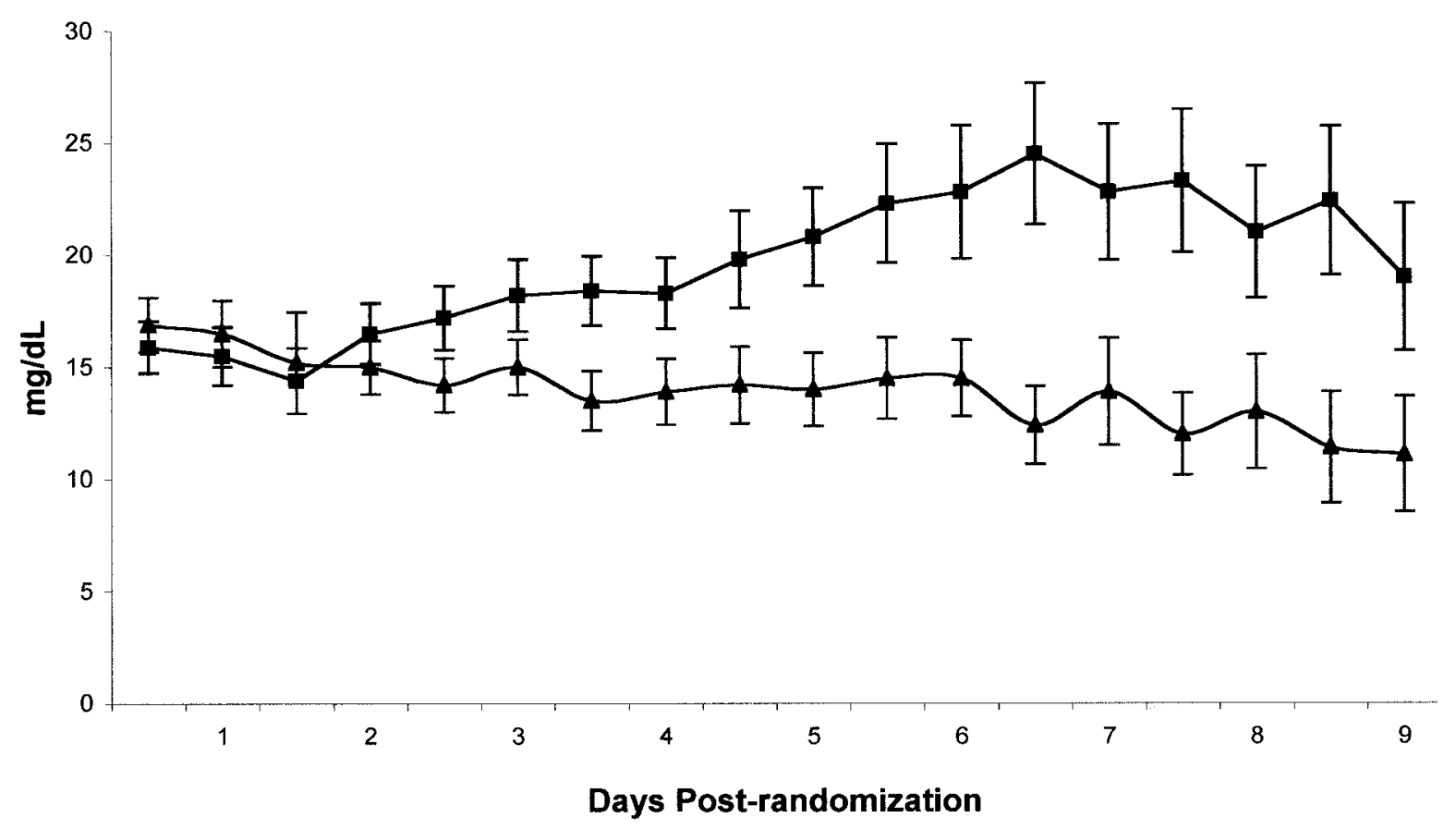

FIGURE 3. Total serum bilirubin levels (mean $\pm S D$ ).

analysis was unlikely. When confounding factors were accounted for in the analysis, there was no statistically significant difference in survival between the 2 groups in the entire patient population. However, when PNF patients were excluded, FHF/SHF patients treated with the BAL had a statistically significant survival advantage compared with controls (44\% reduction in mortality). Data analysis excluding PNF patients is justified because they undergo early retransplantation and derive no benefit from any possible BAL-induced reduction in ICP due to the their chronic disease and absence of intracranial hypertension.

Although previously we reported clinical neurologic improvement, reduction in ICP and increase in cerebral perfusion pressure, as well as reductions in plasma levels of plasma ammonia and serum transaminases after BAL treatment, ${ }^{6,7,9,17}$ in this study, laboratory and neurologic parameters did not reflect beneficial effects. This may be due to differences in sampling times among studies. In the current study, to establish a standardized monitoring regimen for both groups, laboratory and neurologic parameters were determined every 12 hours regardless of BAL treatment time, while in previous studies they were measured at the start and end of each BAL treatment. ${ }^{6,7,9}$

Total serum bilirubin levels in BAL patients were lower compared with controls. This is most likely due to improved liver function as a result of BAL treatment(s); however, increased bilirubin conjugation by porcine hepatocytes and excretion of bilirubin in the bile and urine are also possible. No plasma exchange was carried out, and the charcoal column used in the BAL was cellulose-coated with a low molecular weight cutoff allowing only passage of small peptides.

The mechanism of the BAL effect is not clear. It is possible that even a small increase in functional liver cell mass ( 7 billion hepatocytes/treatment) has a significant effect in patients with minimal residual liver functional mass. Another possibility is that treatment with cells results in profound physiologic effects that enhance liver regeneration and recovery. ${ }^{18}$ The relative contributions of hepatocytes and charcoal in the system need to be defined.

\section{CONCLUSION}

This is the first prospective, randomized, controlled, multicenter trial demonstrating a survival advantage for an extracorporeal liver assist system in FHF/SHF patients. The BAL demonstrated a favorable safety profile. The outcome of the study becomes more important when one takes into account the heterogeneity of the patient population, the large number of centers involved with varying local standards of care, and the high degree of severity of illness of the study population. 


\section{REFERENCES}

1. Kondrup J, Almdal T, Vilstrup H, et al. High volume plasma exchange in fulminant hepatic failure. Int J Artif Organs. 1992;15:669-676.

2. Awad SS, Swaniker F, Magee J, et al. Results of a phase I trial evaluating a liver support device utilizing albumin dialysis. Surgery. 2001;130:354-362.

3. Horslen SP, Hammel JM, Fristoe LW, et al. Extracorporeal liver perfusion using human and pig livers for acute liver failure. Transplantation. 2000;70:1472-1478.

4. Ellis AJ, Hughes RD, Wendon JA, et al. Pilot-controlled trial of the extracorporeal liver assist device in acute liver failure. Hepatology. 1996;24:1446-1451.

5. Mullon C, Pitkin Z. The HepatAssist ${ }^{\circledR}$ Bioartificial Liver Support System: clinical study and pig hepatocyte process. Expert Opin Invest Drugs. 1999;8:229-235.

6. Watanabe FD, Mullon CJ, Hewitt WR, et al. Clinical experience with a bioartificial liver in the treatment of severe liver failure: a phase I clinical trial. Ann Surg. 1997;225:484-491.

7. Detry O, Arkadopoulos N, Ting P, et al. Clinical use of a bioartificial liver in the treatment of acetaminophen-induced fulminant hepatic failure. Am Surg. 1999;65:934-938.

8. Morsiani E, Rozga J, Scott HC, et al. Automated liver cell processing facilitates large-scale isolation and purification of porcine hepatocytes. ASAIO J. 1995;41:155-161.

9. Samuel D, Ichai P, Feray C, et al. Neurological improvement during bioartificial liver sessions in patients with acute liver failure awaiting transplantation. Transplantation. 2002;73:257-264.

10. Lindsay KL. Fulminant hepatic failure. In: Gitnick G, Hollander D, Kaplowitz N, et al, eds. Principles and Practice of Gastroenterology and Hepatology. New York: Elsevier, 1988:1217-1224.

11. Riordan SM, Williams R. Treatment of hepatic encephalopathy. N Engl J Med. 1997;337:473-479.

12. Whitehead J. Overrunning and underrunning in sequential clinical trials. Control Clin Trials. 1992;13:106-121.

13. Whitehead J. The Design and Analysis of Sequential Clinical Trials, revised 2nd ed. New York:Wiley Chichester, 1997.

14. Pitkin Z, Switzer W, Chapman L. An interim analysis of PERV infectivity in 74 patients treated with a bioartificial liver in a prospective, randomized, multicenter controlled trial. Hepatology. 2001;34:249A.

15. Pitkin Z, Mullon C. Evidence of absence of porcine endogenous retrovirus (PERV) infection in patients treated with a bioartificial liver support system. Artif Organs. 1999;23:829-833.

16. Fagan E, Frame D, Stevens C, et al. Short-term survival in acute liver failure (ALF): impact of transplantation on future design of treatment protocol. Hepatology. 2001;34:284A.

17. Arkadopoulos N, Alexandri M, Papadimitriou D. First clinical application of a second generation Hepatassist bioartificial liver support system without charcoal associated with neurological recovery in a fulminant hepatic failure patient. Gastroenterology. 2001;120:A543.

18. Suh KS, Lilja H, Kamohara Y, et al. Bioartificial liver treatment in rats with fulminant hepatic failure: effect on DNA-binding activity of liverenriched and growth-associated transcription factors. J Surg Res. 1999; $85: 243-250$

\section{Discussions}

Dr. J. Michael Henderson (Cleveland, Ohio): This is an excellent study. I would like to congratulate Dr. Demetriou and his colleagues for performing it. How often do we hear papers or discussions here finishing with the slogan of "This really needs or wants a prospective randomized controlled trial"? Well, here you have it. And I think that is one of the most important things about this paper. This is done correctly.
This is a huge undertaking. These are difficult and complex patients. It required 19 centers worldwide to complete this study with the many investigators you saw.

But they found the centers, they found the patients, they got the patients into this trial. And don't sell yourself short on the outcomes of this study. Before you did this - and I don't think anyone else will ever do this particular trial - the question was sitting out there: Is there really a place for these bioartificial liver devices in these groups of patients? And I think you have defined the groups of patients in whom this is a worthwhile procedure.

The data as I hear it being presented show that the artificial liver confers no advantage to primary nonfunction. And you come up with the reasons and rationale for that. This is a different group of patients who do not have the severe intracranial problems of some of the acute liver failures. That is important data. You have defined that this group of patients probably should not receive this type of liver support.

You show the survivor advantage of patients with acute and subacute fulminant hepatic failure. And when you really get into the detail of it, it is those with the definable etiologies, the bile hepatitis, the acetaminophen poisoning, and some of the known drug-induced liver failures. I think you defined very clearly a group of patients who do benefit from this with very definite survival advantage. So my questions really evolve around the following: Your data in acetaminophen terminated the trial on the basis of their futility analysis and it is really on the subsequent subgroup analysis that you come up with the survival advantage for the fulminant and subfulminant patients with definable etiologies. Are you and your co-investigators truly comfortable with advocating that this group of patients is the only group of patients who should receive the bioartificial liver support when they come in with acute fulminant failure?

My second question revolves around the learning curves and the cost of setting up these systems. You had 19 centers - again, as I said in the beginning, a huge undertaking. How many of these centers were doing this before you did the trial? How complex was it for them to set up the systems? What would you and your co-investigators advocate as the model which should be used in this country and in Europe? How many centers should really do this? Patients presumably should really come into centers with experience with this device. And I wonder what your recommendations are at the end of this study.

My third question relates to your other parameters outcomes. You have previously shown the significant improvement in the cerebral hemodynamics in this group of patients and in some of the other biochemical parameters. You didn't show that in this trial. In the manuscript you certainly talk about perhaps this is a function of when these things were measured. But I think you sort of have it tied to careful protocol. And it would seem to me that really what 
you are showing in this study, it is perhaps all the other things, as you point out. These patients are in the intensive care unit, have a thousand other things done as well as the BAL device. Do you really think it is the other things that are more beneficial in managing the cerebral hemodynamics in this group of patients?

Great study. A prospective randomized controlled trial. You and your co-investigators are to be congratulated on its completion.

Dr. RAvi S. Chari (Nashville, Tennessee): I would like to congratulate the authors on an outstanding presentation and I would like to thank Dr. Demetriou for not only giving me a manuscript many weeks in advance but also inviting me to discuss this paper.

This is the latest presentation from the Demetriou group and is another in a series of longitudinal studies seeking to establish the validity and utility of a bioartificial liver in treating the acutely decompensated liver.

Based on their phase 1 results, statistically predicted modeling was used to anticipate enrollment, outcome, and numbers necessary for this trial. The outcomes were monitored by a data safety monitoring board. In fact, it was this board, as Dr. Henderson pointed out, that terminated the study early because it felt that demonstration of a significant effect using the Whitehead triangular test was unlikely.

Nonetheless, when primary nonfunction patients for liver transplantation were excluded from the analysis, there was a statistically significant benefit in survival in the bioartificial liver group compared to the standard of care group. Establishing a benefit with the exclusion of the primary nonfunction was somewhat surprising. It is surprising because the primary nonfunction group underwent early retransplantation.

In the overall analysis, your data indicate that time to transplantation was longer in the bioartificial liver patients - 5 days compared to 3 , as you pointed out — and also in the primary nonfunction group the rate of survival after transplantation was actually $58 \%$ in the bioartificial compared to $75 \%$ in the control group.

This brings up my first question: Is it possible that those patients undergoing BAL in the setting of primary nonfunction were held off from transplantation in the hope of improved liver function? In other words, was the time to transplantation significantly different in the bioartificial group compared to the control group, meaning they should not have been excluded from the final analysis?

Secondly, I have 2 questions about the system itself. The first concerns the technology of your bioartificial liver device. In the manuscript you indicate that the hepatocytes are in suspension. As hepatocytes are epidermal derived cells and require cell-to-cell contact, have you examined your hepatocytes during the perfusion to determine if they have significant function during the process itself?
As an extension of this, as you allude to in the discussion of your manuscript, what steps are you taking to determine the real contribution of hepatocytes to the improved clinical outcome? I bring up this question only because there is a recent body of literature that supports the utility of noncellular based systems using albumin and protein counter perfusion with or without plasmapheresis that reports similar improvements in the setting of acute liver failure.

My last question is more of a futuristic question. Dr. Demetriou, your group has done groundbreaking work in the area of hepatocyte stem cells. Do you see an application of stem cells of human origin in your bioartificial liver in the future?

Dr. William C. Meyers (Philadelphia, Pennsylvania): Over the past 2 decades, Dr. Demetriou has chosen the Southern Surgical Association as his forum for presenting progress in the development of his bioartificial liver technology. We have watched as he has almost single-handedly persisted in turning doubters into believers that liver dialysis is in fact a reality, or could be a reality.

I became a believer at Duke when I helped Dr. Chari, the previous discussant, lead a team to develop a percutaneous whole pig liver dialysis technique that really worked for fulminant liver failure. We watched in amazement as a patient in stage 4 coma with fixed, dilated pupils and being considered for brain death, awakened enough to sign an OR permit for liver transplantation. Likewise, Dr. Demetriou has now demonstrated numerous clinical successes with his bioartificial liver.

I compare this technology with coronary artery bypass grafting in its clinical infancy, when that procedure clearly worked yet it was still difficult to demonstrate survival benefits beyond all statistical doubts. Dr. Demetriou now has developed an excellent product that will continue to be improved.

The success of this product now will be primarily driven by business factors. The question has become: Will this be a product driven or a customer driven technology? Ultimate success will depend on it being the latter; that is, being customer driven.

How usable will this product be? Who will be the primary corporate sponsors? Two companies at least have already met a sorry fate with liver dialysis as a primary part of their product lines. How much profit will be in it for the present corporate sponsors?

Dr. Demetriou, would you enlighten us with respect to the market analysis on your product? This product will not have the potential market size of kidney dialysis. Liver dialysis seems unlikely to become, like kidney dialysis, a way to chronically sustain life in the best of circumstances. What is the market size for liver dialysis? Who exactly will be the market? What will be the price of use? And how will this technology be sustained from the standpoint of profitability? 
These are difficult questions, but there must be speculative answers to sustain investment interest. What round of funding presently is this product in right now? Who are the present principal owners of this technology?

Dr. Demetriou, I really do believe in your product. But belief in a practical sense is not really enough. In order to succeed, this product needs to be customer based. How close to the right answer are you with respect to this fundamentally important question?

Dr. Demetriou, congratulations once again. You are one of my heroes. And thank you on behalf of the Southern Surgical Association for choosing us as a forum for presenting your amazing innovations.

Dr. William C. Wood (Atlanta, Georgia): I rise in admiration of this work and this paper. As a clinical trialist I would point out the enormous effort that went into these centers and all these patients, and yet in terms of statistical significance what a really small study this is. Today in oncology we typically will have 2000 to 3000 people in a group in order to detect a clinically beneficial effect. You had to cut the risk almost in half to achieve statistical significance with a group this size. In order to demonstrate an improvement of $30 \%$, which may include all of your patients, it would have taken a much larger trial. So, first, I think we must not underestimate the dramatic size of the benefit you seem to be showing here.

Also as a clinical trialist, I would point out that your experimental design, while interesting, compares the entire treatment with the artificial liver. If these were mice and not people, I would very much have liked to see you compare the plasmapheresis and everything except the hepatocytes to answer how much of the improvement reflects the plasmapheresis and the charcoal filter rather than the true artificial liver.

Dr. Achilles A. Demetriou (Los Angeles, California): The trial was terminated because it was felt that if we were to continue with the 30-day survival endpoint, using the Whitehead triangular testing model without taking into account the dramatic impact of transplantation, we were not likely to see an effect certainly in the first 400 patients. The sample, compared to major pharmaceutical trials, was small and there were a number of factors that worked against us, especially the inclusion of PNF patients. Also, organ allocation policies changed in the first year of the study. The effect was that there were now more organs allocated for fulminant patients around the country and there were more organs shifted into the fulminant liver failure patient population, with resulting earlier transplantation of those patients.

We felt that if we had 5 days, based on an earlier pilot study, to show the efficacy of the system, we could actually do it. But when patients were getting transplanted within 24-48 hours, it was very hard to show a beneficial effect of any treatment during this short window of time. The length of time of allowing access to the treatment to a show a potential beneficial effect wasn't there in the PNF patients.

Dr. Chari asked if the fulminant group is the only one most likely to benefit from treatment. We don't know. What we do know is that in this specific group we did see a beneficial effect.

To answer Dr. Meyers' question, no commercial model can succeed based on treating fulminant patients only. There are just not enough of them. The major opportunity involves treating acute-on-chronic liver failure patients, where you have thousands of patients throughout the world. And those would be the patients where eventually this product would have to be tested in from a commercial development aspect.

There is a learning curve with this device and there is significant cost associated with it. However, the cost of managing the fulminant patient, including the cost of transplantation, is significantly greater than the cost of transplant in an elective setting. Also, the results of long-term survival in fulminant patients are not as good as in elective transplants. So the overall cost of transplantation and managing these patients is actually very significant. If we were able to treat patients and get them to a point where they are not going to need a transplant and they can recover spontaneously, then we will have a model that makes sense not only for the patient but for the provider.

In terms of demonstrating other beneficial effects of BAL treatment, we could not show neurologic improvement because many of the centers that participated in the trial, especially in Europe, would not use intracranial pressure monitors. It would have been impossible for to us demonstrate secondary beneficial effects in both biochemical and neurologic parameters because of the complexity of the trial and treatment variations among the centers.

According to protocol, all patients in the trial should have received a transplant immediately as an organ became available. We cannot exclude the possibility, however, that when patients were placed on the BAL the surgeons felt that something was being done for them especially if they were improving and an organ could be allocated to another more gravely ill patient. But we have no data based on the analysis from the various centers suggesting that this was the case.

Regarding the role of the various BAL components, we have data in the animal setting demonstrating that the best design is one that includes hepatocytes and charcoal. We tested hepatocytes alone, charcoal alone, but the best results in pigs with ischemic liver failure were seen when we had both.

Regarding the question about the role of non-biologic dialysis-based systems, we believe that they may be useful in some patients with very high levels of drugs in their plasma. But I really think that in the long run to sustain liver failure 
patients, there will be a need for provision of both detoxification and synthesis.

And then regarding use of stem cells. We and many others, probably including yourself, are spending a lot of time and effort trying to develop human stem cells, stem-cell derived cells that we can use in future trials. We are not close to having a useful cell line at this time.
Regarding device design, future devices have to be simpler. They have to be adjustable and easily adapted to existing kidney dialysis systems. And they have to be easily introduceable into the clinical setting without training costs, without special technical costs, to make them cost-effective enough to be introduced in the clinical setting. The major clinical use of these devices will be in acute-on-chronic liver failure. 